\title{
Spherical SiGe quantum dots prepared by thermal evaporation
}

\author{
Yu-Cheng Liao, Shih-Yen Lin, and Si-Chen Lee \\ Department of Electrical Engineering, National Taiwan University, Taipei, Taiwan, Republic of China \\ Chih-Ta Chia \\ Department of Physics, National Normal University, Taipei, Taiwan, Republic of China
}

(Received 26 June 2000; accepted for publication 24 October 2000)

\begin{abstract}
SiGe alloy quantum dots with spherical shape have been synthesized by the thermal evaporation method. The shape and structure of these dots have been investigated. Transmission electron microscopy images show that they have an average diameter of about $15 \mathrm{~nm}$, and their cores are crystalline. The composition of these dots could be extracted from the Raman peak position of the silicon TO phonon. (C) 2000 American Institute of Physics. [S0003-6951(00)02752-2]
\end{abstract}

Due to their interesting optical and electrical properties, semiconductor nanoparticles have attracted much attention in recent years. ${ }^{1-4}$ One of the most interesting topics is the synthesis of $\mathrm{SiGe}$ quantum dots. Recently, the popular method to synthesize SiGe quantum dots is using the traditional crystal growth technique such as molecular beam epitaxy (MBE) to growth $\mathrm{SiGe}$ on $\mathrm{Si}$ substrate. ${ }^{4,5}$ The lattice constant difference between $\mathrm{Si}$ and $\mathrm{SiGe}$ leads to the formation of self-organized quantum dots. In 1976, Granqvist and Buhrman ${ }^{6}$ have investigated a method to form ultrafine metal particles in an inert gas atmosphere. Si quantum dots grown by the same method have been investigated by Morisaki et al. in recent years. ${ }^{7,8}$ In this study, the thermal evaporation technique was used to synthesize SiGe alloy quantum dots. The shape and structure of these dots have been investigated by using the transmission electron microscopy (TEM), transmission electron diffraction (TED), and Raman spectroscopy.

The schematic diagram of the growing system is shown in Fig. 1. Si and Ge powders are loaded into different tantalum (Ta) boats in a vacuum chamber. The distance between boats and glass substrate can be adjusted from 8 to $13 \mathrm{~cm}$. In this study, the distance was fixed at $13 \mathrm{~cm}$. The chamber is evacuated to $10^{-4}$ Torr at first, then it is purged with Argon (Ar) gas and evacuated for several times. Finally, the chamber is refilled with Ar to the pressure about 0.1-10 Torr. With the glass substrate cooled to liquid nitrogen temperature, the Ta boats are heated to the desired temperature. The $\mathrm{SiGe}$ dots are formed in the Ar gas by collision induced growth and eventually deposited on the substrate. Finally, the liquid nitrogen was removed and when the specimens were warmed to room temperature, they were taken out from the work chamber.

The TEM images of nanoparticles grown by this method were investigated. Figures 2(a), 2(b), and 2(c) display the bright-field, dark-field TEM image and TED pattern of SiGe quantum dots grown at a pressure of 1 Torr, where the boats temperature were set at 1500 and $1400{ }^{\circ} \mathrm{C}$ for $\mathrm{Si}$ and $\mathrm{Ge}$ boats, respectively. This specimen is called S64. It is found the core of the dot all brighten in the dark-field TEM image but have a smaller size than that observed in the bright field. This tells us that the dot have a single crystal structure in the core and surrounded by a thin oxide shell with thickness about $2.5 \mathrm{~nm}$. The average dots size is about $15 \mathrm{~nm}$. It is also found that some of the particles are darker than others. With the previous discussion, it is thought that these dots are deposited on the substrate randomly, so they have different diffracting orientations which can cause the dark contrast in the TEM image. Though the dots look like a sphere in the bright field, but the core seems much more like polyhedron with facets in the dark field. This possibly depends on the difference between the oxidized rates of different facets. This result is direct evidence that the $\mathrm{SiGe}$ alloy quantum dots prepared by this method have an alloy structure but not separate into different parts.

In order to make sure that the structure of these dots was $\mathrm{Si}-\mathrm{Ge}$ alloy but not pure Si dot or Ge dot, the Raman spectrum of SiGe quantum dots shown in Fig. 2 was measured and shown in Fig. 3(a). The Raman spectrum of another specimen called S61 which set the boats temperature to about 1600 and $1400{ }^{\circ} \mathrm{C}$ for $\mathrm{Si}$ and Ge boats, respectively, was shown in Fig. 3(b). Three peaks are found in the spectra, which correspond to different vibration modes in the dots. In Fig. 3(a), the peak locations are 464, 288, and $396 \mathrm{~cm}^{-1}$, which correspond to TO phonon of silicon germanium and $\mathrm{Si}-\mathrm{Ge}$ alloy, respectively. The TO phonon peak of $\mathrm{Si}$ shifts from 521 to $464 \mathrm{~cm}^{-1}$ because of the content of Ge in the dots is high. Alonso and Winer ${ }^{9}$ have studied the relationship between the peak location and $\mathrm{Ge}$ content in the SiGe bulk which could be written as

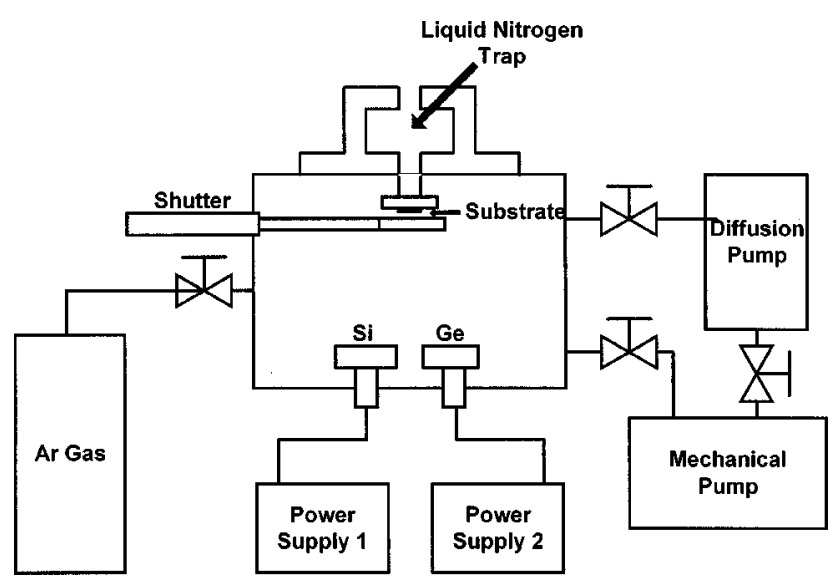

FIG. 1. The schematic diagram of the growing system. 


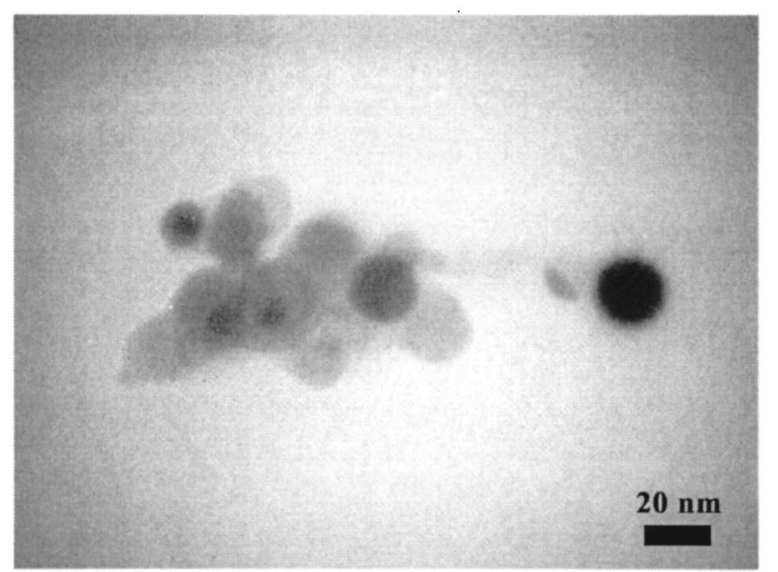

(a)

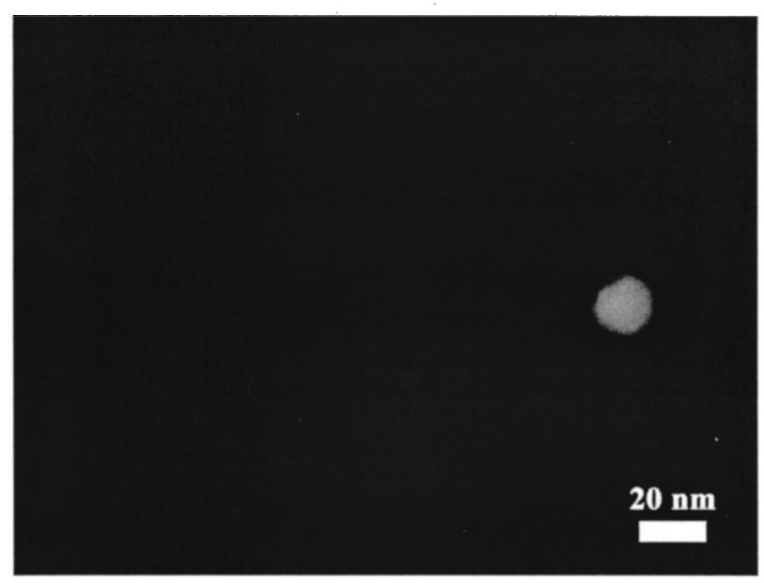

(b)

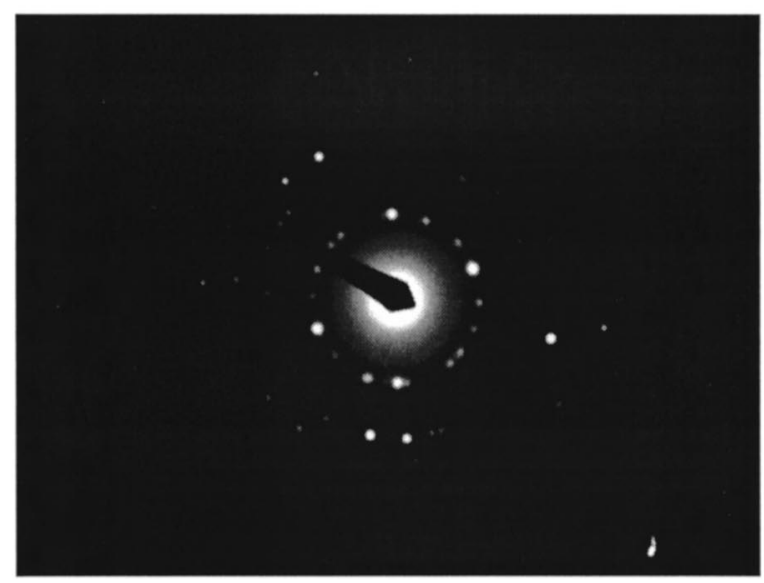

(c)

FIG. 2. (a) Bright-, (b) dark-field TEM image of specimen S64, and (c) the TED pattern of dots shown in (a).

$$
y=520-70 x,
$$

where $y$ is the peak position and $x$ is the fraction of Ge in the bulk. Based on this result, it could be estimated that the fraction of Ge in specimen S64 is near 0.8. And Ge fraction in specimen S61 is about 0.3. It shows that the dots formed in the Ar gas is of the structure of crystalline $\mathrm{Si}-\mathrm{Ge}$ alloy, and the composition of these dots could adjusted by varying the temperature of different boats.
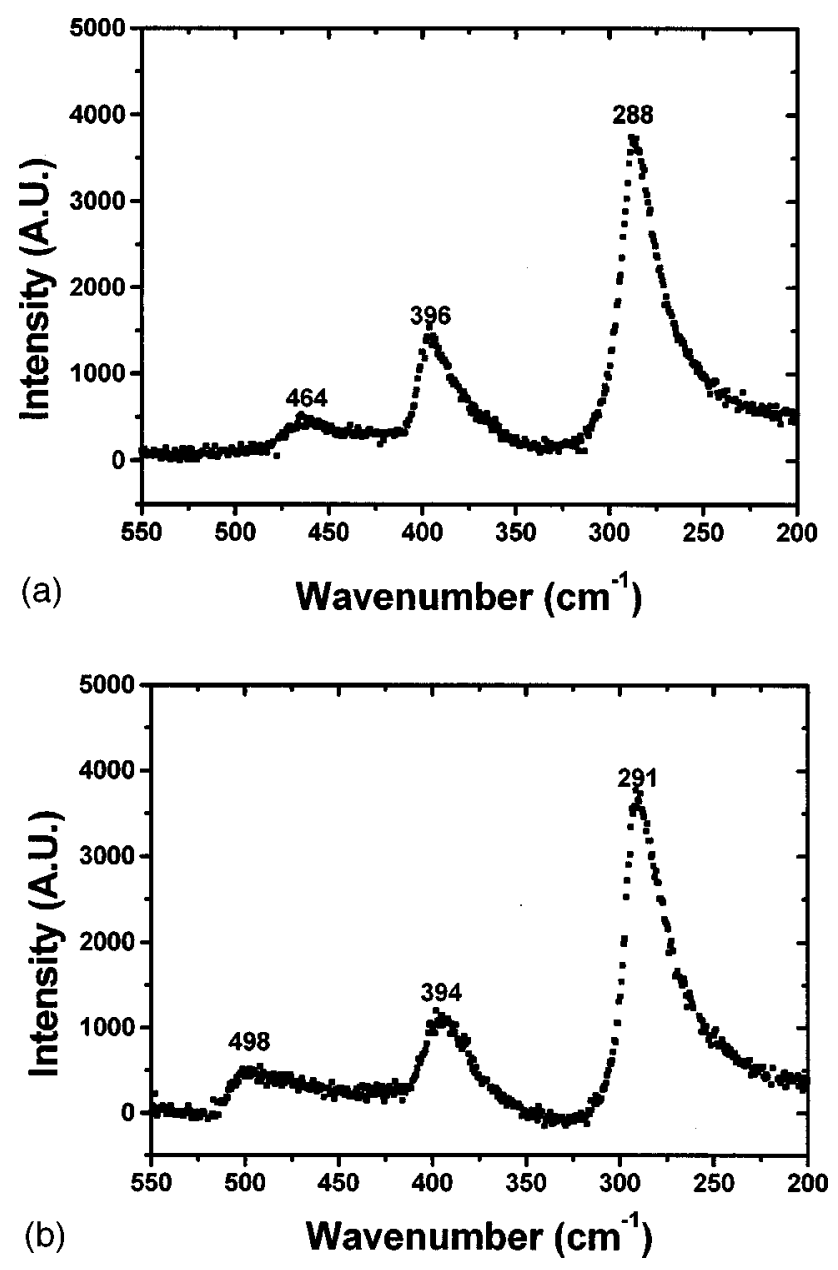

FIG. 3. Raman spectra of specimens (a) S64 and (b) S61.

Finally, it is concluded that thermal evaporation technique has been applied to synthesize SiGe quantum dots with spherical shape. The composition of these dots could be adjusted simply by varying the applying current to different boats. This method is much simpler than the present MBE technology and has a high potential in optical application.

This work is supported by National Science Council of the Republic of China under Contract No. NSC89-2215-E002-018.

${ }^{1}$ V. Craciun, I. W. Boyd, A. H. Reader, and D. E. W. Vandenhoudt, Appl. Phys. Lett. 65, 3233 (1994).

${ }^{2}$ H. Chen, W. Q. Cheng, X. G. Xie, Q. Huang, and J. M. Zhou, Appl. Phys. Lett. 70, 446 (1996)

${ }^{3}$ L. Harris, D. J. Mowbray, M. S. Skolnick, M. Hopkinson, and G. Hill, Appl. Phys. Lett. 73, 969 (1998).

${ }^{4}$ Y. S. Tang, C. M. Sotomayor Torres, W. X. Ni, and G. V. Hansson, Superlattices Microstruct. 20, 505 (1996).

${ }^{5}$ P. Schittenhelm, G. Abstreiter, A. Darhuber, G. Bauer, P. Werner, and A. Kosogov, Thin Solid Films 294, 291 (1997).

${ }^{6}$ C. G. Granqvist and R. A. Buhrman, J. Appl. Phys. 47, 2200 (1976).

${ }^{7}$ H. Morisaki, F. W. Ping, H. Ono, and K. Yazawa, J. Appl. Phys. 70, 1869 (1991).

${ }^{8}$ H. Morisaki, H. Hashimoto, F. W. Ping, H. Nozawa, and H. Ono, J. Appl. Phys. 74, 2977 (1993).

${ }^{9}$ M. I. Alonso and K. Winer, Phys. Rev. B 39, 10056 (1989). 